\title{
Augmenting Performance: Vocal Activity Compared to Presence in the Social Facilitation of Running
}

\author{
Humza Malik ${ }^{1}$ \\ ${ }^{1}$ Santa Clara High School, Santa Clara, CA, USA
}

\section{$\underline{\text { ABSTRACT }}$}

Objective The impact of encouragement on performance has been observed in the setting of exercise, but further research is necessary in running. To compare the improvement in pace experienced by subjects of different backgrounds (from a running team, non-running sports team, or no sports team) as a result of active (verbal encouragement) and passive social facilitation, this study was conducted.

Method Eighteen male high school students, age $17.3 \pm 0.75$ years were recruited for this study, six from each background listed above. Participants were randomly assigned to one of three treatments: external verbal encouragement (EVE), without external verbal encouragement (WEVE), or no presence. Improvement in pace after the introduction of a social facilitator was recorded along with overall consistency in pace. Statistical significance was set at $\alpha=0.10$.

Results A significant improvement in short-term pace among subjects from a running team was generated by EVE, $t(1)=-5.198, p=0.0605$, and no presence generated a significant improvement in short-term pace among subjects from a non-running sports team, $t(1)=-5, p=0.0669$. EVE also generated a significant improvement in long-term pace among subjects from a running team, $t(1)=-5.069, p=0.0620$. A conclusion on consistency was not reached.

Conclusion Verbal encouragement saw to long-term and short-term improvement in pace of subjects selected from a running team, while the lack of presence assisted the short-term performance of subjects selected from non-running sports teams. Results obtained offer potential to impact competitive long-distance running.

\section{Introduction}

Humans are intrinsically social creatures; they crave connections and share much of the behavior of social animals, including more complex forms of affiliation and the creation of hierarchy (Young, 2008). In 1943, an analysis of human behavior by social psychologist Abraham Maslow allowed him to establish the existence of five human needs, termed the "Hierarchy of Needs." Following physiological and safety needs in this model, Maslow theorized that humans will seek relationships that satisfy their need to belong, and respect to satisfy their need for esteem (Maslow, 1943). The human need for affection and respect play into two of the behavioral tendencies that tend to characterize social animals: affiliation ${ }^{1}$ and the establishment of hierarchy. Socialization is essential; in fact, social isolation and the lack of the activity, has far-reaching consequences, ranging from a faster rate of cognitive decline to poorer cognitive performance and executive function (Cacioppo and Hawkley, 2009).

With the social nature of humans, it is not that astonishing then that they are influenced by the presence of others (Allport, 1924/1975). In this paper, one aspect of the influence that humans have upon others shall be explored: social facilitation, the process by which the completion of a task or activity by an individual is made easier by social presence. While the effect of social presence on others is seemingly definite, in reality it varies based on where it is

\footnotetext{
${ }^{1}$ the desire of social animals to be a part of groups
} 
observed. In the case of tasks like multiplication, social presence improves one's ability to complete the task (Allport, 1924/1975). Conversely, tasks involving new learning, and not the application of past skills, can be impeded by the presence of other individuals (Husband, 1931; Pessin, 1933). This paper aims to identify how social presence impacts high school running.

\section{Literature Review}

\section{Positive Impacts of Social Presence}

While the existence of social facilitation in competitional sports had been hypothesized prior to the 20th century, research on this process only emerged near the start of the last century, and this field continues to branch off to this day. Social facilitation was first observed in a formal setting in the 1898 cycling experiments conducted by psychologist Norman Triplett (Strube, 2005). Triplett observed the times produced by cyclists in unpaced, paced, and competitive races, and his analyses established the fact that both competitional racing and cycling accompanied by pacers resulted in a reduction in the pace of cyclists by 20-30s per mile, a drastic improvement from cyclist pace when they were not being observed (Strube, 2005).

The process was given a name in 1924, after researcher Floyd Allport published a study noting significant differences between individuals who were in experimental groups and those who participated in an experiment alone in tasks like multiplication (Allport, 1924/1975). Allport observed an increase in the speed and quantity of work produced by those who were in experimental groups, but results could only be generally attributed to social facilitation, as in reality social relationships in groups are more complex than they were in the experimental settings (Allport, 1924/1975). The general idea behind this is well-known; oftentimes, the pressure of a spectating person or crowd can urge one to exceed limits and to some degree, perform better.

\section{Negative Impacts of Social Presence}

However, the impact of social presence on performance is not only positive; in certain cases, social presence can impede the completion of a task. The detrimental impact of social presence was illustrated in a study of finger-maze learning, where blindfolded subjects were required to trace through a maze (Husband, 1931). Later, social presence was found to impede performance again in the learning of nonsense syllables (Pessin, 1933). In both cases, by introducing a social presence factor, subjects encountered greater difficulty in completing the tasks and time necessary for completion was increased.

Through combining prior research, another psychologist, Robert Zajonc, published a new set of findings, attributing the improvements in performance experienced as a result of social facilitation to increased arousal, and concurrently discovering the limits to which social facilitation was observed (Zajonc, 1965). He concluded that when one is participating in an activity they are familiar with, social facilitation tends to be observed, but when one participates in an activity they are unfamiliar with or must learn, social presence can actually hinder performance (Zajonc, 1965). Much of Zajonc's findings remain unchallenged and applicable to this day.

\section{Social Facilitation and Running}

The impact of social facilitation on running and other sports has been a field of recent exploration. Many researchers have been eager to uncover how passive and/or active facilitation can impact one's performance in running. This has been explored in two scenarios in particular: short-distance and long-distance running.

In an examination of both short-distance and long-distance running, it was found that active social facilitation caused a significant difference in subject performance in running trials, via experimentation conducted on untrained 
but active individuals (Edwards et al., 2018). Verbal encouragement was found to bolster the performance of the subjects in both endurance and sprint exercises, suggesting the positive impact of active social facilitation on running of those who are not competitive runners (Edwards et al., 2018).

In experimentation on long-distance running alone, research demonstrated the lack of impact of peer-based social facilitation (Carnes, 2011). In an evaluation of how a peer impacted the running of intercollegiate distance runners, runners were either paired to run on treadmills or told to run alone. The research concluded that the social presence brought by a peer had no impact on their running. However, it was suggested that the lack of effect may have been attributed to the indoor setting and the reduced competitiveness that ensued, and the potential that the elite nature of the distance runners may have reduced the need for social facilitation (Carnes, 2011).

While social facilitation of running has been studied, no studies addressed this topic as it applied to teenagers, those from different levels of familiarity with the sport, and with a comparison of active (with vocal encouragement) and passive social facilitation. In an attempt to further understand the process of social facilitation as it applied to this age range and under these circumstances, a question surfaced: "Does vocal social activity have a comparable difference to mere social presence in affecting a high school runner's times in the $800 \mathrm{~m}$ run at a self-declared pace?"

\section{Hypothesis}

It was initially hypothesized that social facilitation would be observed with both passive and active facilitation and for all selected students, regardless of current sports participation, but most significantly in students bolstered with verbal encouragement who did not participate in a sport. High school students are required to complete a certain quantity of running, and it was assumed that this amount of running would be sufficient to familiarize students with running such that improvement would be observed for all students. Students who did not participate in a sport were expected to be most impacted by social facilitation, as it was believed that their lack of sports activity would cause their inability to control their pace following the introduction of a facilitator. This hypothesis was created as a result of past conclusions on social facilitation, which showed statistically significant improvements in ability while one was in the presence of others and while completing tasks of familiarity (Strube, 2005; Allport, 1924/1975; Zajonc, 1965; Edwards et al., 2018).

\section{Methodology}

In order to test the hypothesis, an inquiry of the pretest-posttest control group design was implemented. The pretestposttest control group design allows the creation of both an experimental and a control group, with a pretest completed before application of the treatment, and the posttest completed after. Students were selected based on their alignment with one of three strata: participation on a running team, participation on a non-running sports team, or nonparticipation, after which they were exposed to a limited amount of information regarding the expectations of the study along with informed consent forms. Following the agreement of subjects, they were randomly assigned to one of three treatment groups.

\section{Participants}

The population of interest for this study was the student body of a large suburban high school in California, and the sample was selected from this population. It consisted of 18 male students, mean \pm SD: age $17.3 \pm 0.75$ years. Those selected from the high school running team self-identified as being trained (in accordance with team regimentation), physically active individuals, while participants selected based on their involvement on a non-running sports team self-identified as being untrained in running, while still physically active. Those who did not participate in a sports team claimed to be physically inactive. 


\section{Treatments}

Two treatments were named and designed to align with use in a study published in 2018 in the BMJ Open Sport \& Exercise Medicine (Edwards et al., 2018): the external vocal encouragement (EVE) and without external vocal encouragement (WEVE) treatments. In both the EVE and WEVE conditions, the social facilitator was hidden for the duration of the first half of the running distance and introduced only at the start of the second half, where they stayed present for the remainder of the trial.

\section{Eternal Verbal Encouragement (EVE)}

The EVE condition was characterized by a single male social facilitator exhibiting active social facilitation, in which positive vocal encouragement (i.e. phrases such as "You're doing great!", "Keep up that pace!", and "Just one more lap!") was used on subjects at the start of the second half of the running distance. Verbal encouragement was provided by the social facilitator for the fifth 100-meter stretch of the 800 meter distance.

\section{Without External Verbal Encouragement (WEVE)}

The WEVE condition was characterized by the same male social facilitator but exhibiting passive social facilitation. The social facilitator followed the same procedure as in the EVE condition and was introduced at the start of the second half of the running distance. However, under this condition, only spectatorship but no vocal encouragement was utilized.

\section{No Presence}

Subjects under the no presence condition were isolated for the duration of the experimentation. No social presence, with the exception of the researcher to time and instruct the patient, was allowed. The researcher was kept distanced from the subject in the hopes of minimizing the impact of social presence. This treatment was designed to serve as a control for the experiment.

\section{Procedure}

Prior to conducting any trials, all prospective participants were contacted, after which they were presented with an informed consent form (see Appendices $A$ and $B$ ). The consent form included a description of the physical expectations, while not disclosing the actual goal as to not invalidate the proposed research. Any who opted to not participate in the study were replaced with others who met the same selection criteria to ensure a final total sample size of 18 students. After obtaining the consent of all selected participants, the study was initiated.

For all trials, the track was cleared, with the only social presence being the researcher, the single subject, and a facilitator if applicable. Subjects were positioned at the starting line of a standard outdoor athletic track (see Figure 1 at end of section), 400 meters in length, that had marker cones at every 100 meter checkpoint of the track (i.e. 100, 200 , and 300 meters from the starting line of the track, with one at the starting line itself). Once positioned, they were instructed on the expectations of the study. Subjects were asked to run two laps on the track, a total distance of 800 meters, at the fastest pace they could manage while maintaining consistency throughout. Prior to starting, participants were reminded that their data would be confidential and that although results from the study may be released publicly, none of the data produced would be published with their name. In order to protect the results from skew that could be caused as a result of a subject's prior awareness of the social presence factor, subjects who were to receive vocal encouragement or passive social facilitation were left uninformed that a facilitator would be introduced.

A smartphone stopwatch was used in the timing of participants. After instructing a subject on the study expectations, a countdown was initiated, after which the stopwatch was started, and the subject was signaled to begin running. Following the completion of every 100 meters of the total 800-meter distance, identified by a subject crossing 
the placed marker cones, time was lapped. After each subject's trial was completed, they were debriefed, and the true purpose of the study was disclosed.

Lastly, time measurements taken during the trial were logged on a spreadsheet. For data analysis, three components of the subject time were analyzed: the short-term and long-term change following the introduction of a social facilitator and consistency in pace. All determinations of significance were made with the use of a t-test for mean difference.

The calculation of significance for the short-term change was obtained by taking the fifth 100-meter stretch of the running distance (directly following the completion of the first lap) and using the percent change in pace:

$$
\text { percentchangeinpace }=\frac{\text { fifth100meterstretch }-100 \text { metersplitaverage }}{100 \text { metersplitaverage }} \cdot 100
$$

In the analysis of long-term change, the percent lap differential was used for comparison:

$$
\text { percentlapdifferential }=\frac{\text { secondlaptime }- \text { firstlaptime }}{\text { firstlaptime }} \cdot 100
$$

Lastly, for consistency, the mean standard deviation of the eight 100-meter subjects ran by each subject was compared between treatment groups.

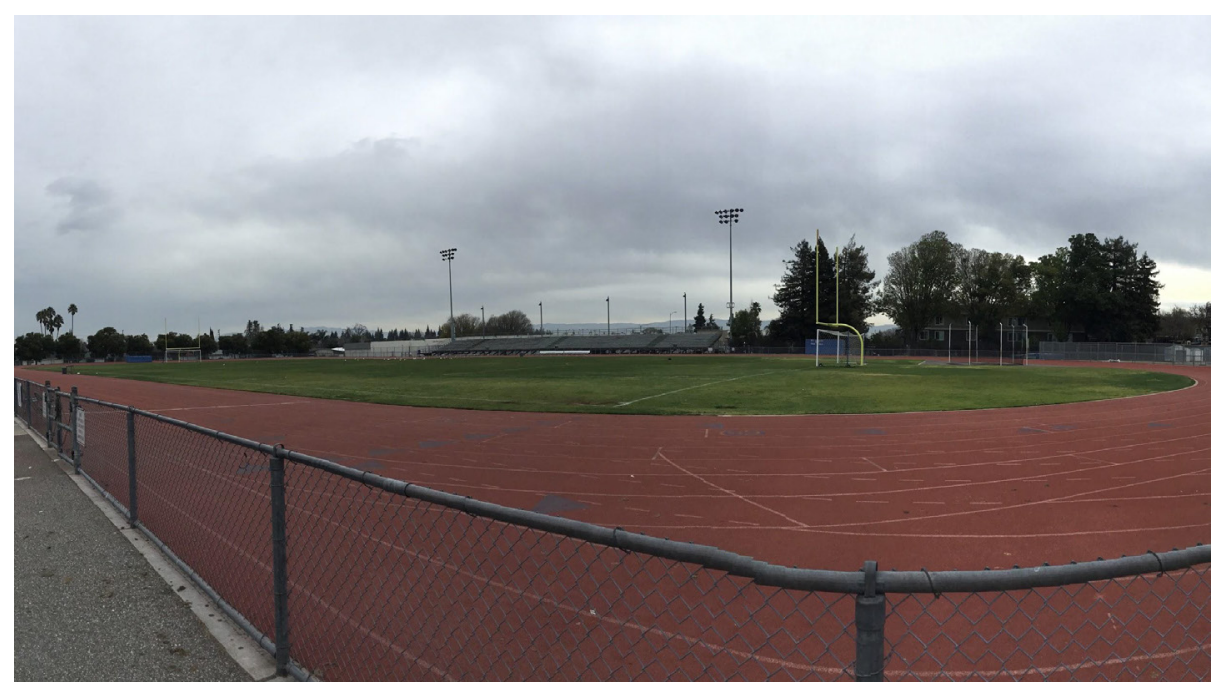

Figure 1. Setting for experimentation

\section{Findings}

The results of this study include an analysis of three factors of the 800 meters run by subjects: short-term change following the introduction of a social facilitator (except in the control group), long-term change following the introduction of a social facilitator (except in the control group), and overall consistency, calculated from the standard deviation and range of time recordings taken following the completion of each 100 meter stretch. This necessitated the calculation of the percent change in pace over the fifth 100 meter segment (after social facilitation was introduced for non-control groups) relative to the subject's segment mean, the lap differential as a percent, and the standard deviation of the eight segments run by each subject. Percentages were utilized to standardize data and avoid disproportionate analysis caused by varying subject average pace. 


\section{Short-term Change}

\section{0m Percent Change from 100m Average}

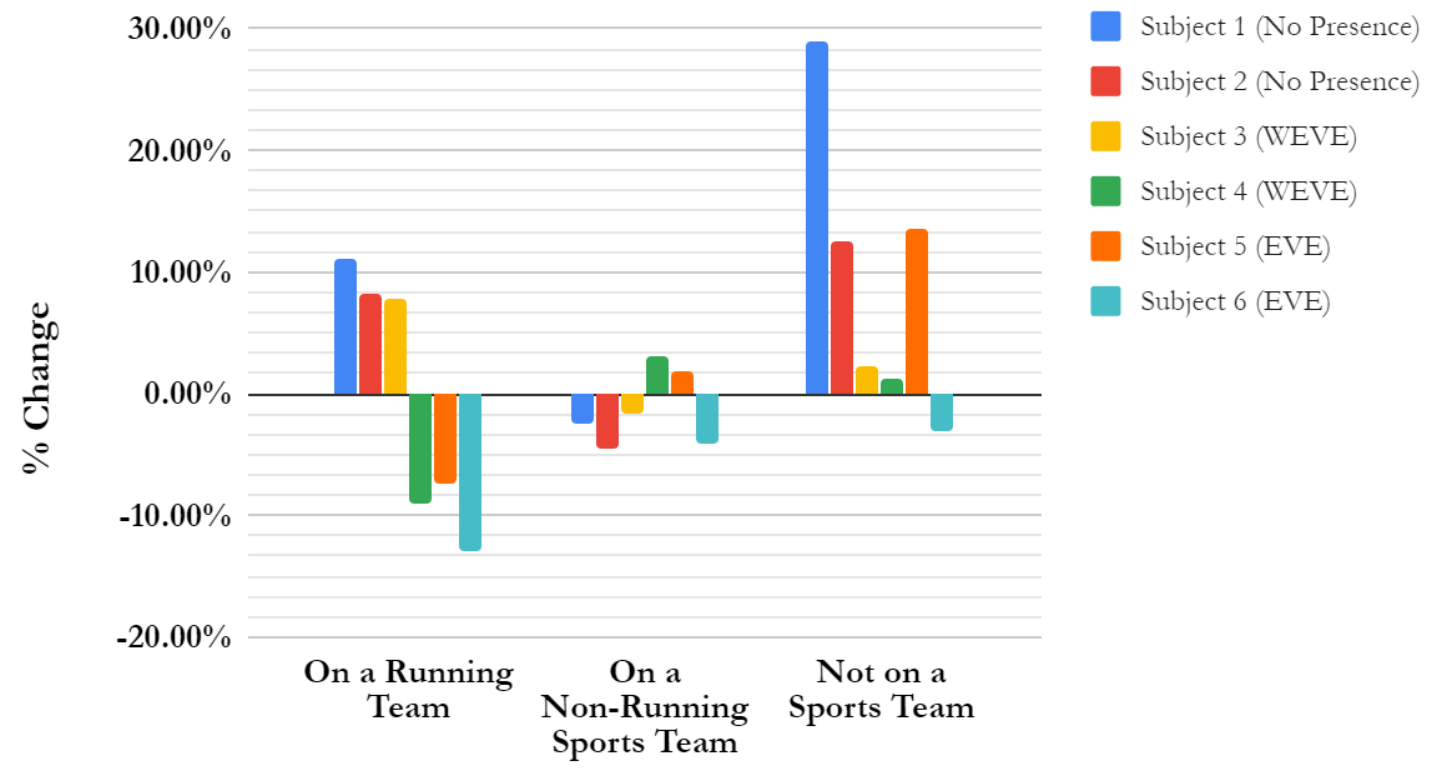

Figure 2. Percent change of the fifth out of eight segments of the distance run, relative to the mean segment speed.

Subjects were graphed based on the stratum grouped in: on running team, on non-running sports team, or not on sports team, and the experimental treatment administered: no social presence, without external vocal encouragement (WEVE), or external vocal encouragement (EVE). Subject numbering is relative to each stratum.

To identify momentary changes caused by the introduction of a social facilitator, the 800-meter distance the subjects ran was split into eight equal-sized segments. The percent change in pace of the fifth segment, directly following the introduction of a social facilitator, was calculated to determine the presence of convincing evidence that facilitation was observed. Short-term increase was noticed to be significantly different from the assumed population norm of 0 in two scenarios: under the condition of EVE, selected subjects from a running team experienced an improvement in pace $(\mathrm{M}=-10.1 \%, \mathrm{SD}=0.028), t(1)=-5.198, p=0.0605$, and under the no presence condition, selected subjects from a non-running sports team also experienced a short-term improvement in pace $(\mathrm{M}=-3.50 \%, \mathrm{SD}=$ $0.011), t(1)=-5, p=0.0669$. The data suggests that EVE facilitates the short-term running of those on a running team, and isolation facilitates the short-term running of those on a non-running sports team.

Significance Tests:

$$
\begin{aligned}
& \alpha=0.10 \\
& \mathrm{H}_{0}: \mu=0 \\
& \mathrm{H}_{\alpha}: \mu<0
\end{aligned}
$$

On a Running Team, EVE: $\mathbf{p}=\mathbf{0 . 0 6 0 5}<\boldsymbol{\alpha}$

On a Running Team, WEVE: $\mathrm{p}=0.4715 \geq \alpha$

On a Running Team, No Presence: $\mathrm{p}=0.9666 \geq \alpha$

On a Non-Running Sports Team, EVE: $\mathrm{p}=0.3445 \geq \alpha$

On a Non-Running Sports Team, WEVE: $\mathrm{p}=0.6311 \geq \alpha$

On a Non-Running Sports Team, No Presence: $\mathbf{p}=\mathbf{0 . 0 6 6 9}<\boldsymbol{\alpha}$

Not On a Sports Team, EVE: $\mathrm{p}=0.8904 \geq \alpha$ 
Not On a Sports Team, WEVE: $\mathrm{p}=0.9389 \geq \alpha$

Not On a Sports Team, No Presence: $\mathrm{p}=0.9215 \geq \alpha$

Overall, EVE: $\mathrm{p}=0.2901 \geq \alpha$

Overall, WEVE: $\mathrm{p}=0.5694 \geq \alpha$

Overall, No Presence: $\mathrm{p}=0.9480 \geq \alpha$

\section{Long-Term Change}

\section{Lap Time Percent Differential Between Laps}

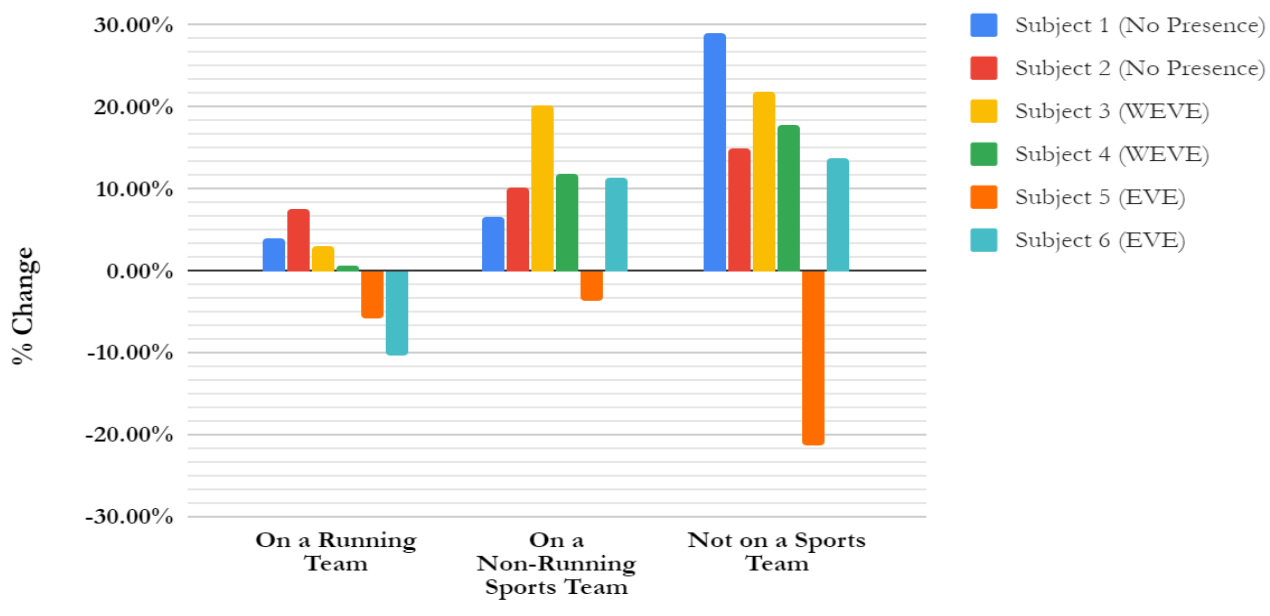

Figure 3. Lap differential between Lap 2 and Lap 1 as a percent. Participants were graphed based on the stratum grouped in: on running team, on non-running sports team, or not on sports team, and the experimental treatment administered: no social presence, without external vocal encouragement (WEVE), or external vocal encouragement (EVE). Subject numbering is relative to each stratum.

To identify lasting changes caused by the introduction of a social facilitator, the percent change in pace of the second half of the run, directly following the introduction of a social facilitator, was calculated to determine the presence of convincing evidence that facilitation was observed. The introduction of a social facilitator was found to cause an increase in pace $(\mathrm{M}=-8.00 \%, \mathrm{SD}=0.022)$ significantly different than the estimated difference of 0 only for subjects selected who were on a running team and subjected to EVE, $t(1)=-5.069, p=0.0620$. The long-term change in pace in other experimental groups was not found to be significant, even under the condition of EVE. This suggests that only EVE has long-term benefits in running and only for those on a running team.

Significance Tests:

$$
\begin{aligned}
& \alpha=0.10 \\
& \mathrm{H}_{0}: \mu=0 \\
& \mathrm{H}_{\alpha}: \mu<0
\end{aligned}
$$

On a Running Team, EVE: $\mathbf{p}=\mathbf{0 . 0 6 2 0}<\boldsymbol{\alpha}$

On a Running Team, WEVE: $\mathrm{p}=0.8629 \geq \alpha$

On a Running Team, No Presence: $\mathrm{p}=0.9326 \geq \alpha$

On a Non-Running Sports Team, EVE: $\mathrm{p}=0.6991 \geq \alpha$

On a Non-Running Sports Team, WEVE: $\mathrm{p}=0.9434 \geq \alpha$

On a Non-Running Sports Team, No Presence: $\mathrm{p}=0.9530 \geq \alpha$

Not On a Sports Team, EVE: $\mathrm{p}=0.4062 \geq \alpha$

Not On a Sports Team, WEVE: $\mathrm{p}=0.9768 \geq \alpha$

Not On a Sports Team, No Presence: $\mathrm{p}=0.9293 \geq \alpha$ 
Overall, EVE: $\mathrm{p}=0.3069 \geq \alpha$

Overall, WEVE: $\mathrm{p}=0.9934 \geq \alpha$

Overall, No Presence: $\mathrm{p}=0.9918 \geq \alpha$

\section{Consistency}

Table 1. Range and standard deviation of the eight $100 \mathrm{~m}$ stretches that were a part of the $800 \mathrm{~m}$ every subject was asked to run, sorted by stratum subject was placed in and treatment administered: no social presence, without external vocal encouragement (WEVE), or external vocal encouragement (EVE)

\begin{tabular}{|c|c|c|c|c|}
\hline Stratum & Subject & Treatment & Range & $100 \mathrm{~m} \mathrm{SD}$ \\
\hline \multirow{6}{*}{$\begin{array}{l}\text { Running Team } \\
\text { (Stratum 1) }\end{array}$} & $\mathrm{A} 1$ & No Presence & 3.91 & 1.56 \\
\hline & A2 & No Presence & 3.61 & 1.24 \\
\hline & A3 & WEVE & 3.96 & 1.29 \\
\hline & A4 & WEVE & 4.14 & 1.46 \\
\hline & A5 & EVE & 4.14 & 1.24 \\
\hline & A6 & EVE & 5.30 & 1.76 \\
\hline \multirow{6}{*}{$\begin{array}{l}\text { Sports Team } \\
\text { (Stratum 2) }\end{array}$} & $\mathrm{B} 1$ & No Presence & 4.04 & 1.32 \\
\hline & $\mathrm{B} 2$ & No Presence & 4.97 & 1.88 \\
\hline & B3 & WEVE & 13.38 & 4.19 \\
\hline & B4 & WEVE & 5.23 & 1.87 \\
\hline & B5 & EVE & 7.46 & 2.36 \\
\hline & B6 & EVE & 6.99 & 2.23 \\
\hline \multirow{6}{*}{$\begin{array}{l}\text { No Sports Team } \\
\text { (Stratum 3) }\end{array}$} & $\mathrm{C} 1$ & No Presence & 12.81 & 4.61 \\
\hline & $\mathrm{C} 2$ & No Presence & 5.96 & 2.20 \\
\hline & $\mathrm{C} 3$ & WEVE & 10.20 & 3.62 \\
\hline & $\mathrm{C} 4$ & WEVE & 7.43 & 2.49 \\
\hline & $\mathrm{C} 5$ & EVE & 23.67 & 8.93 \\
\hline & $\mathrm{C} 6$ & EVE & 8.60 & 3.14 \\
\hline
\end{tabular}


Differences in mean standard deviation between conditions were found to be insignificant at a significance level of $\alpha$ $=0.10$. Despite observable differences in standard deviation, trials would have to be repeated with a greater sample size or differences would have to be more noticeable in order to draw any conclusions of significance.

\section{Mean Standard Deviation:}

Overall, EVE: 3.276 seconds

Overall, WEVE: 2.489 seconds

Overall, No Presence: 2.134 seconds

Significance Tests:

$$
\begin{aligned}
& \begin{array}{l}
\alpha=0.10 \\
\mathrm{H}_{0}: \mu_{1}-\mu_{2}
\end{array}=0 \\
& \mathrm{H}_{\alpha}: \mu_{1}-\mu_{2}>0 \\
& \quad \text { EVE }- \text { WEVE: } \mathrm{p}=0.2719 \geq \alpha \\
& \quad \text { EVE - No Presence: } \mathrm{p}=0.1948 \geq \alpha \\
& \text { WEVE - No Presence: } \mathrm{p}=0.3151 \geq \alpha
\end{aligned}
$$

\section{Discussion}

This study's main purpose was to analyze the impact of two factors on the pace of a subject over a distance of 800 meters. The first of these factors was sports team participation, classified based on three categories: running team participation, non-running sports team participation, and no sports team participation. The second factor was the treatment subjects received: social facilitator with external vocal encouragement (EVE), social facilitator without external vocal encouragement (WEVE), or no presence. The study found that the presence of a male social facilitator providing vocal encouragement had a long-term positive effect on the performance of male members of a high school running team similar to the individuals selected. Additionally, short-term improvement in pace was observed on two instances: in male high school subjects selected from a running team who were subjected to vocal encouragement from the same male social facilitator and in high school subjects not on a sports team who ran in an isolated setting.

To the researcher's knowledge, this marks the first study that confirms both the short-term and long-term impact of positive active social facilitation by a male facilitator on the long distance running of 16-18 year olds from a running team similar to those selected in the study. This may help give insight on how to enhance the performance of runners who compete on running teams over long distances. Findings may be applicable to team running environments in which crowds are exhibiting passive facilitation. When subjects from a running team experienced passive social facilitation, no significant improvement in pace was found. Thus, this study emphasizes the potential of vocal encouragement in the social facilitation of high school runners to encourage improvement.

It may be important to note the short-term improvement of subjects selected from a non-running sports team who ran under the "No Presence" condition. In this setting, subjects produced data that yielded a p-value found to be significant with the used significance level of $\alpha=0.10$. Thus, the increase in pace of selected subjects from a nonrunning sports team in an isolated setting was a noteworthy observation of this study. This hints at a couple different possible conclusions that can be made regarding this improvement, aside from the possibility that increases in pace were due to chance alone. Firstly, in accordance with the research of social psychologist Robert Zajonc, social facilitation is typically observed in instances where the individual being facilitated is familiar with the activity being performed, lest social facilitation may actually impede the completion of an activity (Zajonc, 1965). As such, the lack of presence of a social facilitator in an unfamiliar activity may have allowed subjects to experience significant improvements starting their second lap, rather than the expected decline. Contrarily, another possible reasoning behind improvement regards the presence of the researcher. While distant from the subject, timing was performed by the 
researcher closest to this segment of the distance, which may have obscured the observation of hypothesized results under this condition.

A conclusion regarding how active and passive social facilitation shaped consistency in a subject's pace could not be drawn. It was hypothesized that the use of vocal encouragement by the social facilitator would result in the greatest variability between the 100 meter segments a subject ran, and that the lack of presence of a social facilitator would result in lower subject pace variability. While this held true for the sample of students taken, with a difference of 0.787 seconds between the mean standard deviation of subjects who experienced EVE and those who were exposed to a social facilitator WEVE, and a difference of 0.355 seconds between the mean standard deviations of subjects who were exposed to a social facilitator WEVE and subjects who lacked a social facilitator, results were not significant at a significance level of $\alpha=0.10$. As such, while this study hints at the existence of a phenomenon, in order to conclude that vocal encouragement and no social presence cause for the greatest and least variability in pace, respectively, more trials would need to be conducted.

\section{Limitations}

Perhaps the most glaring limitation to this research was the difficulty in making conclusions of significance due to the limited sample size. Few students were selected to participate in the study and there was strict selection criteria, which restricted sample size. The small size of the sample only allowed the testing of each treatment within each stratum on 2 subjects, which limited tests of significance run on the data. Achieving significance of the data was only possible at a high significance level $(\alpha=0.10)$, which indicates a 10\% probability that results were due to chance alone. Increasing the size of the sample would allow the reduction of the significance level due to lower p-values which would accordingly decrease the probability of improvements being the result of chance as well. In future studies, expansion of the sample size of the study may help strengthen or refute conclusions made by this study.

Beyond sample size, another limitation of this study regards its use of an irregular basis of selection. Rather than using standardized measurements of weekly hours invested into running or other sports to stratify the population when attempting to select participants for the study, subjects were only selected based on their participation, or lack thereof, on running/sports teams. In reality, mere participation on a sports team does not accurately reflect on the fitness of individuals or the effect that spectatorship may have on them, and thus causes for irregularity when used as a basis for subject selection. This, in accompaniment with the small sample size, makes the generalization of the results uncovered by this study to the population of interest difficult.

\section{Conclusion}

At the start of the study, the aim was to to obtain data suggesting at least three things: the presence of a noticeable effect of active social facilitation on high school student running, differences between the effect social facilitation has on those from different backgrounds and with different degrees of comfortability with running, and differences in the consistency in pace between treatment groups. Following experimentation, the study was able to conclude that vocal encouragement benefits high school members of running teams similar to those selected in the study in both shortterm and long-term measures of pace. Thus, two of the aims, establishing the effect of active social facilitation on running performance and recording differences in how social facilitation affected the running of those from different backgrounds, were accomplished by the termination of the study.

While the findings of this study are limited in application, they still offer promise to settings similar to running meets and other competitional running events, in which performance may be enhanced-in both the short and long term-with the use of active social facilitation. In an attempt to boost the running performance of competitive and/or team runners, vocal encouragement is a feasible and promising solution that can easily be augmented to facilitate improvement. On another track, physically active individuals at a similar level of fitness as the high school 
athletes on a non-running sports team selected in the study may find that they experience better running workouts when in a setting lacking any social presence.

\section{Future Directions}

To further explore social facilitation and more specifically, how it applies to running, the following experiments should be considered. First, a repeat of this experiment, but with a larger sample size and a more thorough, standardized selection. While this study was able to come to statistically significant conclusions, in order to obtain data with lesser probability that data obtained were produced as a result of chance, a larger sample size would be beneficial. Furthermore, selection in this study did not use the optimal criteria for stratification of the population; while the categorical classifications of participation on a running team, participation on a non-running sports team, or lack of participation on a sports team were partially effective in illustrating the levels of fitness and type of exercise performed by individuals selected, quantitative classifications may have been more effective. Variables for selection could include quantities such as hours spent in cardiovascular exercise or average mileage on foot per week.

Other future experiments could regard the use of verbal encouragement and social facilitation in a comparison of precision ${ }^{2}$ (e.g. golf, shooting sports, billiards, etc.) and non-precision sports (typically team sports like basketball, soccer, etc.). Competitive long-distance running is most typically an example of a team, non-precision sport; a different form of concentration and focus is required for success in the sport. The effect of social facilitation and greater crowd spectatorship has manifested in many non-precision team sports and is experimentally known: in the five year period studied by Carron and Paradis, the increased spectatorship witnessed at home games allowed winning percentages for these games of " $53.7 \%$ in baseball, $61.0 \%$ in English football (soccer), 54.6\% in ice hockey, 58.2\% in American football, and 61.0\% in basketball," (Carron and Paradis, 2014). However, studies have yet to place these aforementioned sports in juxtaposition with precision sports under similar conditions to observe how the effects of vocal encouragement in particular, but those of social facilitation in general vary between these different categories of sports. While some of the effects of vocal encouragement on precision sports may be anecdotally known, further experimentation is necessary to substantiate many claims on the impact of this encouragement.

\section{References}

Allport, F. H. (1975). Social psychology. New York: Johnson Reprint. (Original work published 1924)

Cacioppo, J. T., \& Hawkley, L. C. (2009). Perceived social isolation and cognition. Trends in cognitive sciences, 13(10), 447-454.

Carnes, A. (2011). The effect of peer influence on running speed, enjoyment, and perceived exertion in intercollegiate distance runners (Doctoral dissertation, Kent State University).

Carron, A., \& Paradis, K. (2014). The home advantage. Encyclopedia of sport and exercise psychology. Thousand Oaks (CA): Sage Publications, 301-355.

Edwards, A. M., Dutton-Challis, L., Cottrell, D., Guy, J. H., \& Hettinga, F. J. (2018). Impact of active and passive social facilitation on self-paced endurance and sprint exercise: encouragement augments performance and motivation to exercise. BMJ open sport \& exercise medicine, 4(1), e000368.

${ }^{2}$ sports requiring accuracy or precision of their players, typically with the use of targets, holes, or other 
Husband, R. W. (1931). Analysis of methods in human maze learning. Journal of Genetic Psychology, 39(2), 258277.

Maslow, A. H. (1943). A theory of human motivation. Psychological Review, 50(4), 370-396.

Pessin, J. (1933). The comparative effects of social and mechanical stimulation on memorizing. The American Journal of Psychology, 45(2), 263-270.

Strube, M. (2005). What Did Triplett Really Find? A Contemporary Analysis of the First Experiment in Social Psychology. The American Journal of Psychology, 118(2), 271-286.

Young S. N. (2008). The neurobiology of human social behaviour: an important but neglected topic. Journal of psychiatry \& neuroscience: JPN, 33(5), 391-392.

Zajonc, R. B. (1965). Social facilitation. Science, 149(3681), 269-274. 